\title{
Current Challenges and Perspectives in Resistive Gaseous Detectors: a manifesto from RPC 2012. ${ }^{1}$
}

\author{
Diego Gonzalez-Diaz \\ Laboratorio de Física Nuclear y Altas Energías, Zaragoza, Spain. \\ Department of Engineering Physics, Tsinghua University, Beijing, China. \\ GSI Helmholtz Center for Heavy Ion Research, Darmstadt, Germany. \\ E-mail: diegogondunizar.es
}

\section{Archana Sharma}

CERN, Physics Department, Geneva, Switzerland.

E-mail: archana.sharmalcern.ch

\begin{abstract}
Resistive gaseous detectors can be broadly defined as those operated in conditions where virtually no field lines exist that connect any two metallic electrodes sitting at different potential. This condition can be operationally recognized as 'no gas gap being delimited by two metallic electrodes' [1]. Since early 70's, Resistive Plate Chambers (RPCs) are the most successful implementation of this idea, that leads to fully spark-protected gaseous detectors, with solid state -like reliability at working fields beyond $100 \mathrm{kV} / \mathrm{cm}$, yet enjoying the general characteristics of gaseous detectors in terms of flexibility, optimization and customization.

We present a summary of the status of the field of resistive gaseous detectors as discussed in a dedicated closing session that took place during the XI Workshop for Resistive Plate Chambers and Related Detectors celebrated in Frascati, and especially we review the perspectives and ambitions towards the XII Workshop to be celebrated in Beijing in year 2014. Due to the existence of two specific reviews $([1,2])$ also at this workshop, a minimum amount of overlap was found to be unavoidable. We have realized, however, that the three works provide a look at the field from different optics, so they can be largely considered to be complementary. Contrary to the initial concerns, the overall appearance seems to be fairly round, in our opinion.
\end{abstract}

Disclaimer: authors have tried being as faithful as possible to the original comments, as well as providing a proper 'wrapping up' for the less educated reader. The session was recorded and it might be made available from the organizers, so that the expert reader can judge on his own.

XI workshop on Resistive Plate Chambers and Related Detectors (RPC2012)

INFN-Laboratori Nazionali di Frascati, Italy

February 5-10, 2012

\footnotetext{
${ }^{1}$ Text based on discussions between attendants to the workshop and a panel formed by S. Bianco, P. Dupieux, P. Fonte, G. Iaselli, A. Musso, S. K. Park, R. Santonico, V. Peskov.
} 


\section{Introduction}

More than twenty five years have passed after the conception of the Large Hadron Collider [3], back in late 80's. At the moment of writing, at CERN, Geneva, the LHC is dramatically pushing our frontier of knowledge to energy regimes never explored before. This is bringing not only tantalizing hints in the long-awaited Higgs sector [4], the dense and hot partonic phase expectedly formed in very energetic heavy ion collisions is also being studied with higher-than-ever precision, as well as CP-violation. There is an ongoing search for evidence of super-symmetric particles that might explain the dark matter needed by our current cosmological model, alongside a number of Standard Model extensions and other exotica.

Resistive gaseous detectors under the standard form of Resistive Plate Chambers (RPCs) $[5,6]$ play a fundamental role in this global scientific breakthrough, due to their high-end timing properties, reliability, ability to easily scale to large areas and magnetic field immunity. Amongst other advantages, that are partly addressed in this document, they offer a rational option for the fast triggering and tracking of muons over large areas (for a review see [7]).

The technological branching that took place in the second half of the 90's [8,9] has sped up the dissemination of the RPC technology into two main directions: i) classic single-gap RPCs like the ones constituting the trigger systems of CMS and ATLAS [10,11] (an optimal and welldeveloped choice for the coverage of large areas when aiming at scintillator-like reliability, magnetic field immunity and $\sim$ ns time-of-flight tagging at high efficiency), and ii) multi-gap timing RPCs for particle identification purposes like the ALICE TOF-barrel [12] (offering also high reliability and magnetic field immunity, but intrinsically more versatile and capable of providing 50ps-accurate time-of-flight tagging at high efficiency). ${ }^{2}$

From the early days of the Pestov counter back in 1971 [5], there has been indeed a large diversification and growth of the RPC field, especially once the technology developed by the soviet school was adapted to commercial materials and made workable at atmospheric pressures, a crucial step accomplished by Santonico and Cardarelli in 1981 [6]. The term 'Resistive Plate' was hence coined, giving birth to the 'Resistive Plate Chamber' (RPC) technology. This breakthrough was to result in a slow but steady democratization of the usage of gaseous parallel plate configurations worldwide. Some consequences of this evolution we can see today, exemplified by the broad range of resistive-plate choices, the universal operation at atmospheric pressure, and the reduction of the initially very extreme operating conditions down to a much more comfortable (yet extreme) saturated-avalanche mode, with a large part of the amplification load moved into the Front End Electronics. The advent of the multi-gap

${ }^{2}$ At the present stage, RPCs are more naturally classified from a structural point of view as 'wide gap' $(\sim 1 \mathrm{~mm})$ or 'narrow gap' $(<0.3 \mathrm{~mm})$, or even functionally as 'trigger' or 'timing', depending on the context, [1]. We follow in this document a technological classification, so as to acknowledge the technical merit of the RPC [5,6], multi-gap [8] and timing [9] developments. There is little ambiguity at the moment, though, whatever adjective is used. 


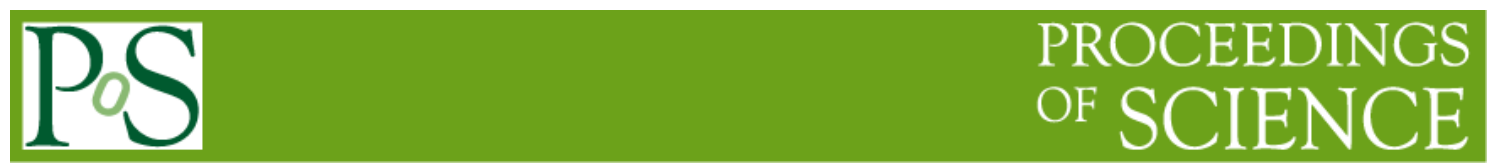

configuration in 1996 [8] finally allowed to technologically 'decouple' the requirements for efficiency and time resolution, thus allowing to obtain excellent timing and efficiency even at atmospheric pressure. Technical and practical problems apart, the time resolution is a matter of the gap characteristics, while a multiple gap can always ensure enough creation of primary ionization clusters, through a procedure that is nowadays known to be easily scalable. Not having found yet the point where these two ideas conflict, the ultimate timing limits of the RPC technology remain widely open (see [13], for instance).

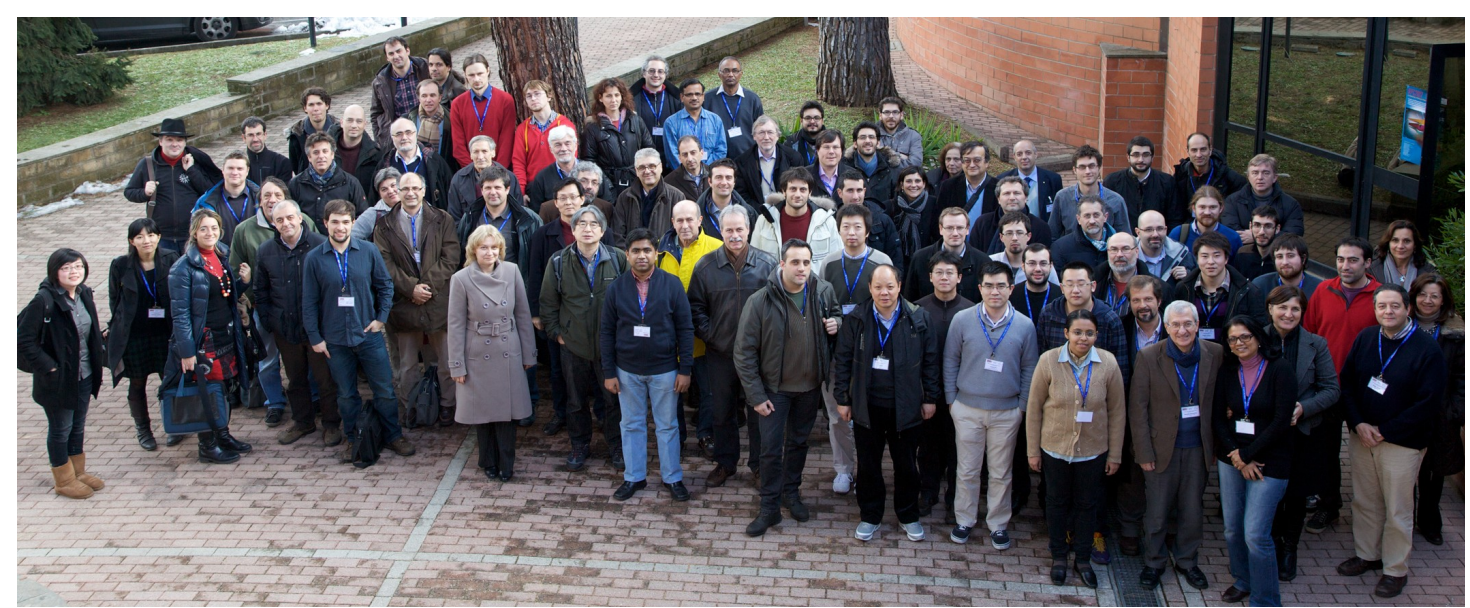

Figure 1: Official photo (detail) of the attendants to the XI Workshop on Resistive Plate Chambers and Related Detectors (courtesy of Claudio Federici).

During the arrangements for the organization of the XI Workshop on Resistive Plate Chambers and Related Detectors (Fig. 1) it was felt that, in view of the recent developments and growth of the RPC technology, a comprehensive compilation of the status and the main future challenges in the field was needed and, with that purpose, the present contribution was born. It is organized along sensitive items discussed during the workshop closing session, which took place around a Discussion Panel constituted by experts in the field and that was moderated by the authors, as follows:

1. Introduction.

2. Status of classic and multi-gap timing RPCs in running experiments.

3. Gas-related ageing, gas chemistry, gas availability and usability.

4. Rate capability, material research and modeling, long-term stability of the electrical properties of the resistive plates.

5. Electronic developments. Discrete electronics and general-purpose ASIC-based amplification/discrimination boards. High accuracy Time to Digital converters.

6. Status and future of simulations.

7. Future of classic and multi-gap timing RPCs.

8. New applications.

9. New trends in Resistive gaseous detectors.

10. Conclusions. 


\section{Status of classic and multi-gap timing RPCs in running experiments}

\subsection{Classic RPCs}

Being originally conceived about two decades ago, CMS and ATLAS sub-systems based on classic RPCs are, however, not first of a kind. Fully fledged large-scale physics experiments, importantly the B-factories BaBar [14] and Belle [15], used them way before LHC started producing its first scientific output. Both CMS and ATLAS RPCs, each extending over a breathtaking area of $4000 \mathrm{~m}^{2}$, have removed all doubt concerning the technologically subtle process of linseed oil coating, which is needed for low dark current and stability of operation of classic RPCs. Fig.2-left shows a 3D-view of the intrinsic efficiency of one of the CMS barrel wheels as obtained for proton collisions at a center of mass energy of ${ }_{s}=7 \mathrm{TeV}$. Values well above $95 \%$ are comfortably and uniformly reached over the whole system [16]. Efforts currently focus on the purification of the gas system, sophisticated humidity monitoring (needed to stabilize the conductivity of the Bakelite/HPL used as resistive plate), background characterization and on-line E/p compensation. Taking into account the soft dependence of the gain with the reduced field in saturated-avalanche operation, the latest correction can be taken as a good indication of the level of sophistication that the technology is reaching (efficiency after correction is stable with operating time within a remarkable $0.5 \%$, i.e., $1 / 200$ particles [16]).

There has been a recent interest in exploiting the RPC timing down to the one intrinsically expected ( $\sigma_{\mathrm{T}} \sim 2 \mathrm{~ns}$ in ATLAS), in view of the need for extending the RPC capabilities beyond its prime role as bunch-crossing tagger. ${ }^{3}$ This has allowed providing some (restricted) particle identification capabilities, in particular resulting in the suppression of loopers, unphysical beam collisions, and natural background radiation [17]. Despite the progress, there is the belief that the full potential of both CMS and ATLAS RPCs has not being fully exploited yet [18].

On another front, LHC -inspired RPCs running in the absence of streamer quenching ('streamer mode'), hence with a much simplified electronic stage and limited time resolution, provide a very convenient technological solution for particle counting over large areas. Such systems are already making contact with fundamental issues in both astrophysics (ARGOcosmic rays, [19]) and particle physics (OPERA- neutrino oscillations, [20]). Currently, OPERA's efforts focus on providing an independent cross-check of the timing accuracy of the Target Tracker, one of the systematic uncertainties present in the recently observed supraluminal neutrino propagation [21]. ${ }^{4}$ Although belonging to the same family, the $140 \mathrm{~m}^{2}$ ALICE muon spectrometer represents a slightly more sophisticated concept, aimed at tagging the $\mathrm{J} / \psi$ and $\Upsilon$ production in heavy ion reactions via proper $\mathrm{p}_{\mathrm{T}}$ selection in their di-muon decay channel.

\footnotetext{
${ }^{3}$ There are different usages of the concept 'intrinsic resolution' in literature. We refer, in this work, to a value for the time resolution that can be independently obtained under reference conditions where the relevant calibrations are either much simpler or straightforward. It thus represents a reasonable practical limit in light of existing evidence.

${ }^{4}$ At the time of writing, there is unconfirmed independent evidence that the discrepancy, if finally traced back to technical limitations of the experimental setup, is very likely not to stem from the Target Tracker performance.
} 


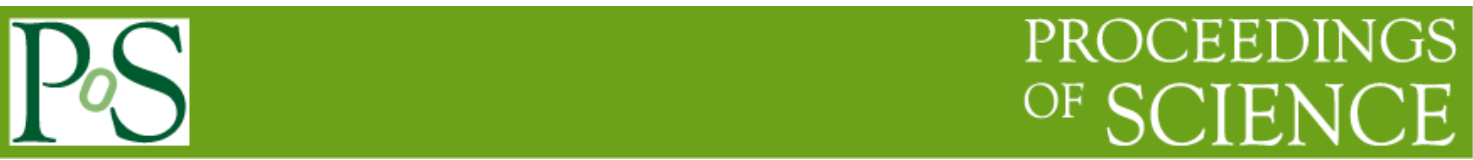

A preliminary analysis of the $\mathrm{J} / \psi$-suppression factor, determined in this way, has been presented at the workshop [22].

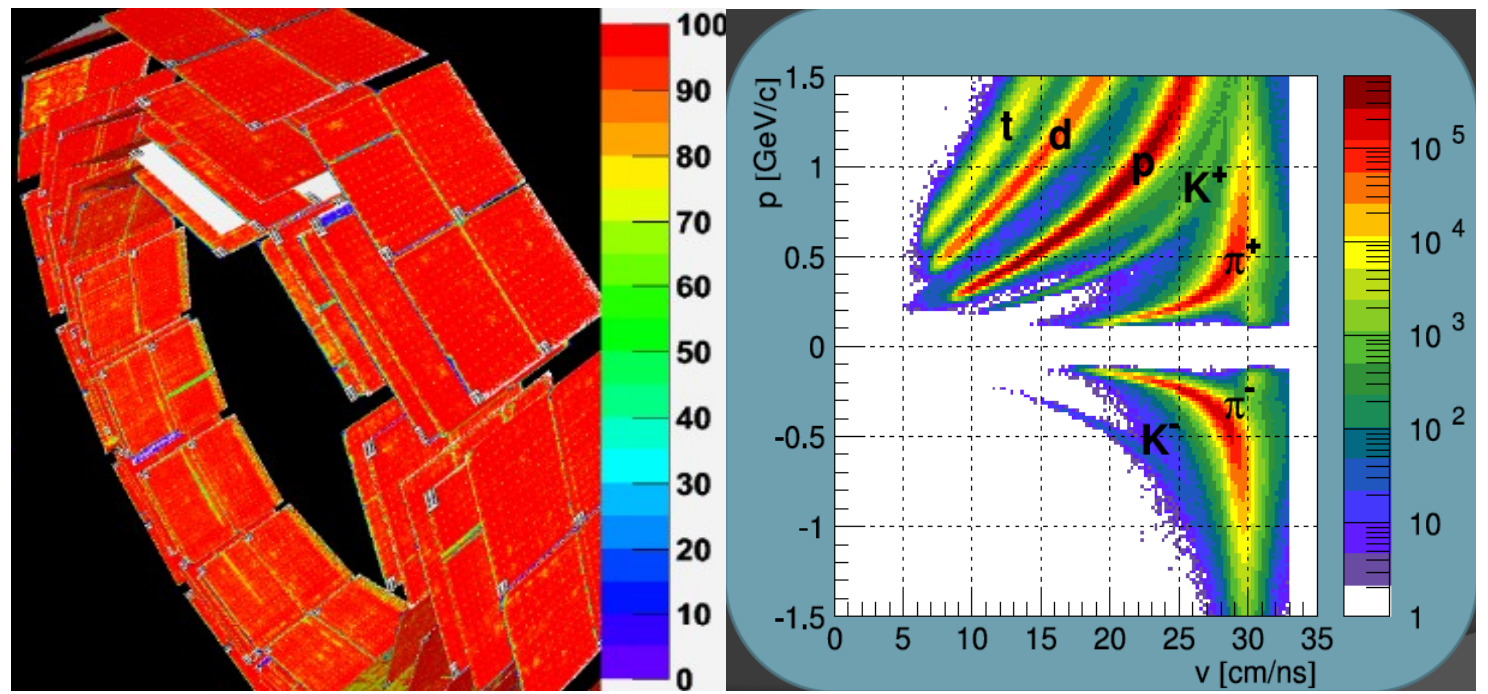

Figure 2: Left: Five radial layers of the classic RPCs constituting one of the CMS barrel wheels, with efficiency indicated on the right axis. Right: particle identification plot from the FOPI experiment as obtained at SIS-18 energies, using multi-gap timing RPCs. $\mathrm{K}^{-}$dynamics sub or close to threshold is a key and difficult observable in heavy ion reactions in the energy regime $\mathrm{E}_{\mathrm{lab}}=1-3 \mathrm{GeV} / \mathrm{A}$; here they are visible to the naked eye. The plot is compatible with an overall system resolution of $\sigma_{\mathrm{T}}=90 \mathrm{ps}$, including the start detector.

\subsection{Multi-gap timing RPCs (MRPCs)}

Pretty much like with classic RPCs, original designs in multi-gap fashion of presently running time-of-flight systems (ALICE[12], STAR[23], HADES[24], FOPI[25]) were conceived more than a decade ago. Fully fledged systems became available and dismantled meanwhile in some singular events, as HARP[26], showing very respectable performances, yet far from the ultimate potential of the technology. Present systems, either with pad [12, 23], multi-strip [25] or single-strip [24] readout, are already capable of comfortably reaching the 100ps landmark and better (see Fig.2-right for a FOPI particle identification plot). Current efforts in HADES and ALICE are focused on the calibrations needed for obtaining, at system level, a time of flight resolution down to the intrinsic value ( $70 \mathrm{ps}$ and $\sim 50 \mathrm{ps}$ for minimum ionizing particles, respectively).

A new MRPC project has recently emerged for large area cosmic ray studies at ground level, within the EEE collaboration. Despite the project has a very strong educational facet, some interesting scientific output has been already produced [27]. The collaboration has managed to obtain 100ps-level resolutions in some counters; however the impact of this performance on the event reconstruction and data analysis has not been explored yet.

In all, there is a general consensus that expectations raised by these new devices, namely, reliable operation over large areas at sub-100ps timing and high efficiency for minimum ionizing particles, have been fulfilled. 


\section{Gas-related ageing, gas chemistry, gas availability and usability}

In view of their large global warming potential (GWP), the usage of two of the main RPC gases, $\mathrm{C}_{2} \mathrm{H}_{2} \mathrm{~F}_{4}$ and $\mathrm{SF}_{6}$, is progressively being banned by authorities ([28], for instance). The future importance of these two gases for the greenhouse effect cannot be neglected since, over a 100 years time-span, predictions give a GWP of 1300 for $\mathrm{C}_{2} \mathrm{H}_{2} \mathrm{~F}_{4}$ while $\mathrm{SF}_{6}$ will exceed 20000 [29]; that, said, means being 20000 more dangerous than $\mathrm{CO}_{2}$. There are two lessons to be learned from the past, based on the fact that these gases are massively used in industrial processes: 1) regulations might be flexible regarding usage for scientific purposes, 2) industry will tend to replace this gas by a functionally equivalent one, expectedly not worse (similar to the $\mathrm{CF}_{3} \mathrm{Br}$ case, [30]). The latter point is even more appealing in the case of $\mathrm{SF}_{6}$ : being one of the best inert electron scavengers known in nature, it therefore exhibits a property that is arguably of comparable importance for RPCs and for industrial HV insulation. Re-circulating systems are definitely an option worth being explored $[29,31]$ although for small R\&D setups they might be not too economical. One could rely on a combination of the two aforementioned hypothetical circumstances (flexibility of regulations and/or industrial replacement) together with a closed loop operation for large systems. Although these options will definitely help avoiding 'sudden extinction' [32], a medium-term strategy seems to be unavoidable.

The most recent exploration of new gas mixtures was carried out by Abbrescia [33], who studied the possibility of adding Helium to the mixture as a 'space holder' gas (in the author's words), i.e., a gas that behaves roughly like a homogeneous vacuum, thus effectively reducing the gas density and consequently the necessary operating field. The principle works, at least qualitatively. However the presence of the standard components of the mixture was respected and just their proportions varied, a fact that does not really help at solving the problem (although it clearly alleviates it, partly). In any case, this effort can be very worthy considered as a first of a new set of experimental studies designed to eventually replace $\mathrm{C}_{2} \mathrm{H}_{2} \mathrm{~F}_{4}$ and $\mathrm{SF}_{6}$ from the standard RPC mixture. Rather complementary, a recent systematic study of the role of both i- $\mathrm{C}_{4} \mathrm{H}_{10}$ and $\mathrm{SF}_{6}$ in timing $\mathrm{RPCs}$ can be found in [34]. It should be stressed that $\mathrm{SF}_{6}$-free operation is definitively possible for timing applications, and both the STAR barrel as well as the future STAR Muon Trigger Detector (MTD) operate in this mode in order to ensure safe operation of the central TPC. Some compromise must be accepted then in terms of a reduced plateau, higher presence of streamers and the subsequent degradation of time resolution. In practice, operation at $90-95 \%$ efficiency with resolutions in the range $90-110 \mathrm{ps}$ under $\mathrm{SF}_{6}$-free gas mixtures has been convincingly demonstrated up to $1 \mathrm{~m}$-long strip counters [35].

Thus, on the one hand, the replacement of both $\mathrm{C}_{2} \mathrm{H}_{2} \mathrm{~F}_{4}$ and $\mathrm{SF}_{6}$ seems to be unavoidable in the medium term. On the other hand, if the gas is to be replaced, ensuring a low ageing for future experiments expecting to deal with life transported charges up to $1-3 \mathrm{C} / \mathrm{cm}^{2}[36,37]$ will require of a systematic and dedicated approach to the problem. Only then it will be possible to guarantee the previously observed low ageing levels present in both classic and multi-gap timing RPCs. At any rate, exploring new gas mixtures while pursuing a microscopic 
understanding and modeling of the gas-related ageing phenomena (along the promising lines in [38], for instance) will certainly require of a multi-disciplinary approach and several years of development, in a realistic scenario.

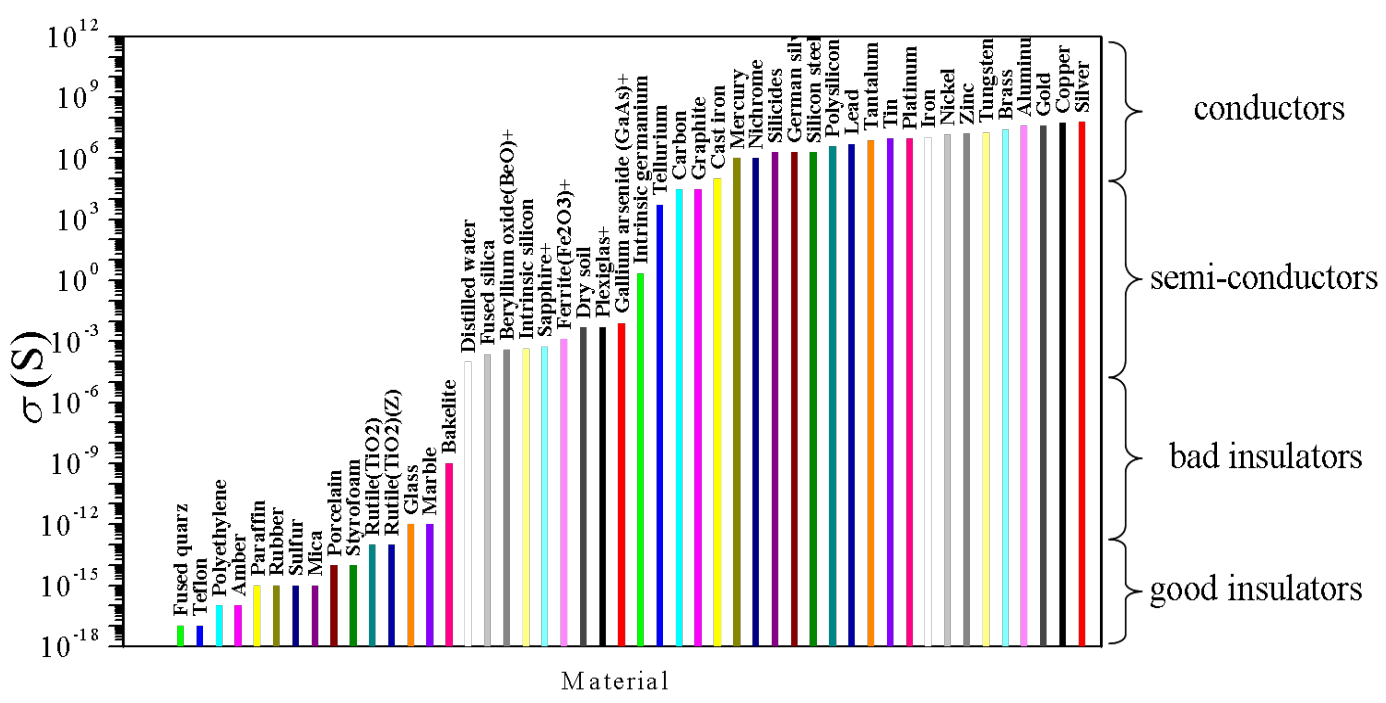

Figure 3: Modified figure from [40] showing a list of popular industrial materials spanning over 30 orders of magnitude. The labeling of two regions as 'bad insulators' and 'good insulators' represent the authors' choice and do not correspond to an universally accepted terminology. The region labeled as 'bad insulators' is the region of interest for RPCs. Over more than 6 orders of magnitude only glass, marble and Bakelite can be found.

\section{Rate capability, material research and modeling, long-term stability of the electrical properties of the resistive plates}

In the immediate future, RPCs enjoying rate capabilities up to $20 \mathrm{kHz} / \mathrm{cm}^{2}$ are necessary both in view of the sLHC upgrade at CERN, Geneva, and of the future SIS-100/300 at FAIR, Darmstadt. By increasing the electronics sensitivity by a factor x5-10 (see next section), operation of classic RPCs has gone down to average charges per gap of around $2 \mathrm{pC}$ in order to fulfill these rate capability ambitions. This is a standard working number for multi-gap timing RPCs ([39], for instance) thus it is not surprising that both CBM MRPCs (at FAIR) and ATLAS classic RPCs are planned at the moment based on materials of similar bulk resistivity $\left(\rho_{20} \sim 10^{10} \Omega \mathrm{cm}\right)$. Despite this remarkable convergence, both materials have a priori a fundamental difference: Chinese doped glass (one of the options for CBM) is polished to ensure the high surface quality required for timing while ATLAS relies on the well established technology of linseed oil -coated Bakelite. General considerations on gas ageing apart (see previous section), ensuring the stability of the electrical properties of the plates is of utmost importance, up to transported charges in the order of $1-3 \mathrm{C} / \mathrm{cm}^{2}$ (i.e., 5 years at the maximum expected flux and $50 \%$ duty cycle) and this has to be consistently demonstrated under realistic conditions. At present, promising results in DC are available for Chinese glass up to $1 \mathrm{C} / \mathrm{cm}^{2}$ and up to $0.05 \mathrm{C} / \mathrm{cm}^{2}$ under X-ray irradiation, showing no sizable effects [37]. On the other hand, since the 
ATLAS-upgrade RPCs will roughly reduce the operating charge per gap by the same amount as the flux will be increased, it can be reasonably expected a low ageing figure, comparable to the one of the already commissioned system.

The challenge of finding new resistive materials was neatly addressed during previous workshop [40]. Roughly speaking, it can be understood as a lack of industrial interest in developing 'bad insulators' (Fig.3), a definition corresponding precisely to the range where both adequate spark-quenching and adequate rate capability can be simultaneously obtained in parallel plate geometries. Besides Bakelite and float glass, materials in the range $\rho \sim 10^{7}-10^{13} \Omega \mathrm{cm}$ have to be custom-developed or are simply rare and thus difficult to find and/or expensive.

In the context of the CBM R\&D for reaching rate capabilities up to $20 \mathrm{kHz} / \mathrm{cm}^{2}$, two new materials have emerged in this 'resistivity gap' and consistent results with prototypes have been shown for already few years: the aforementioned Chinese doped glass developed by Tsinghua University at Beijing [37, 41] with $\rho_{20} \sim 2 \times 10^{10} \Omega \mathrm{cm}$ (ohmic) and the $\mathrm{Si}_{3} \mathrm{~N}_{4} / \mathrm{SiC}$ ceramics developed by the HZDR institute at Dresden [42], with $\rho_{20} \sim 7 \times 10^{9} \Omega \mathrm{cm}$ (varistor-type, tunable). The former has a smooth 'float glass'-like behavior with low dark currents, while the latter, allowing for a higher rate capability, has raised some concerns related to the small signal pickup observed on large-area prototypes as well as large dark currents that are, as yet, unexplained. There are, however, strong indications that the rate capability of the $\mathrm{Si}_{3} \mathrm{~N}_{4} / \mathrm{SiC}$-based counters may comfortably reach the $100 \mathrm{kHz} / \mathrm{cm}^{2}$ landmark under uniform irradiation, at least for small detectors.

Modeling the role of the electrical conductivity of the resistive material in the RPC behavior has been subject of some interest in the past ([43-47]), but a quantitative description of the fundamental process driving the conductivity was never attempted before, to the best of the authors' knowledge. Having such a model in hand will help understanding not only the limitations of certain approaches (in the most typical case, stemming from instabilities appearing as a function of the operating time) but will also help enormously at defining the material requirements when, for instance, contacting partners from the field of material sciences or industry. The well known failure at reproducing Pestov-like glass in Europe should be taken as a warning in this respect. Again, a multi-disciplinary approach has brought the most promising results and a model seems to be now within reach, at least for certain families of materials (Fig. 4). A consistent conductivity model can be expected to describe the behavior with the electric field, temperature and time under the same formalism [48]. Developments in this direction have an enormous potential in applied research $([49,50]$, for instance) and should be prioritized.

In the context of this and previous section, the elaboration of a list of RPC-compatible materials will be increasingly necessary, especially now that operating particle fluxes are going to see a 10 to 100 -fold increase. 


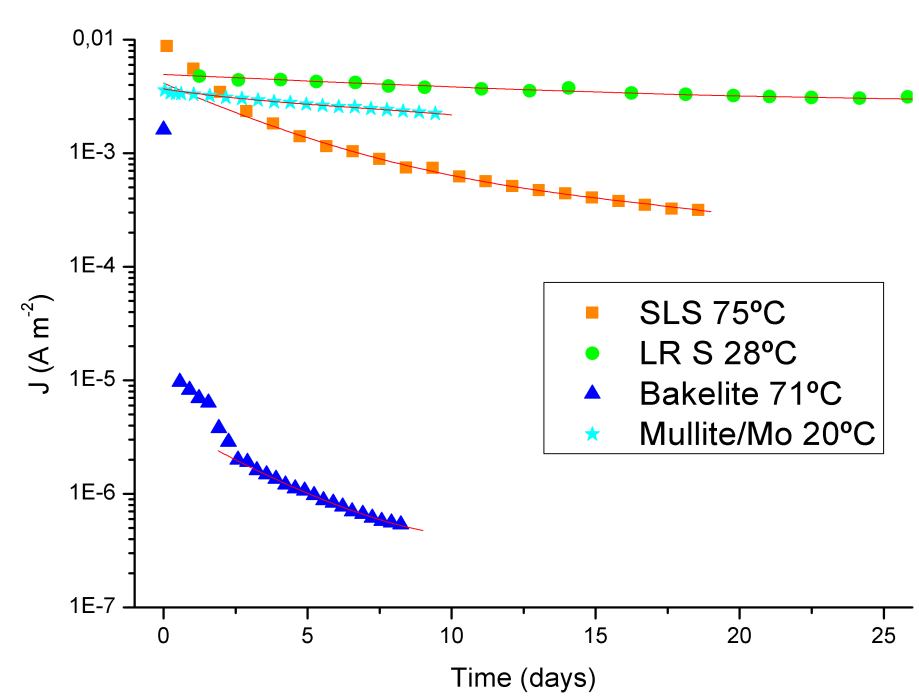

Figure 4: Behavior of the current density flowing across different material samples for a constant voltage $(\sim 1 / \rho)$ for different materials (soda-lime glass, Chinese doped glass, Bakelite and a novel Mullite/Molibdenum ceramic-metal composite). Lines show the behavior based on a conductivity model proposed by Hyde and Tomozawa (1984), (taken from [48]).

\section{Electronics developments. Discrete electronics and general-purpose ASIC-based amplification/discrimination boards. High accuracy Time to Digital converters}

The importance of the Front End Electronics (FEE) was very much stressed at the workshop. This fact obeys a 4-fold reason:

I) First of all, the coverage of large areas with high position resolution (on the order of $100-200 \mu \mathrm{m}$ ) as is typical of applications in medical imaging or homeland security (see section 7) can hardly run with discrete components due to practical limitations as well as power consumption and space. So, the development of ASICs is an appealing direction to go in order to further explore these possibilities. Some fairly general purpose broad-band amplifier/discriminator ASICs like NINO [51] and PADI [52] have been developed and are already under steady use, while a new ASIC, CAD, was presented at the workshop [53].

II) As mentioned in section 4, a reduction on the operating threshold together with the addition of 1-gap ('bi-gap') has allowed the ATLAS detectors to reach operating particle fluxes of $10 \mathrm{kHz} / \mathrm{cm}^{2}$, at an average charge released per gap of around $\sim 2 \mathrm{pC}$, close to the requirements of the ATLAS upgrade [36]. Thus, loosely speaking, increasing the FEE sensitivity has allowed for a $\times 5-10$ reduction in the operating gain (Fig.5-left). This fact results on a power dissipation for $10 \mathrm{kHz} / \mathrm{cm}^{2}$ comparable to the one the present ATLAS RPCs have at the efficiency plateau for a meager $1-2 \mathrm{k} \mathrm{Hz} / \mathrm{cm}^{2}$. As said, the satisfactory ageing figure of the already commissioned system is hence expected not to be worse with the new architecture. This idea of 'moving' gain from the detector to the FEE suggests that thinking of the FEE and RPC as separate entities is not the right way for present and future developments [54]. 
III) It is well known that current amplifiers show significant performance differences as compared to classic charge-sensitive amplifiers [55, 56]. With approximate character, the difference can be traced back to a bandwidth reduction of the later and higher signal to noise ratio. This fundamental difference is, however, not properly addressed by existing simulation works when aiming at a quantitative description of the detector response properties (see next section). Until the electronics and detector are not treated consistently under the same simulation framework, it can be expected that achieving a very necessary global system description will never be fully achieved.

IV) The idea of developing some general-purpose electronics for the RPC community was addressed at the workshop. It was acknowledged that it would be, in general grounds, a very good idea. The key issues are the time jitter $\sigma_{t}=\sigma_{v} / d V /\left.d t\right|_{V=V_{t h}}$ and minimum threshold, say $\mathrm{V}_{\mathrm{th}} \sim 3-4 \sigma_{\mathrm{v}}$. It is unfortunate that the slope at threshold $\mathrm{dV} / \mathrm{dt}$ depends critically on both the signal shape and the amplifier response function while the noise r.m.s., $\sigma_{v}$, and the overall stability are system-dependent. It will take some well-educated systematic study to successfully bridge the later points and come to the specifications of such a general-purpose electronics. On the other hand, it is foreseeable that there will be always special applications that pose special requirements, for instance in terms of multi-hit response (i.e., cross-talk) or signal sensitivity. The complexity of this endeavor, therefore, can not be taken lightly.

Besides Front End Electronics, there has been considerable progress on digitization ASICs, most remarkably the HPTDC [57]. A new FPGA-based board capable of reaching an unprecedented sub-10ps resolution was presented at the workshop [58] (Fig. 5-right), and it will be very interesting to see how this high-precision search evolves and in particular if the RPC technology can catch up.
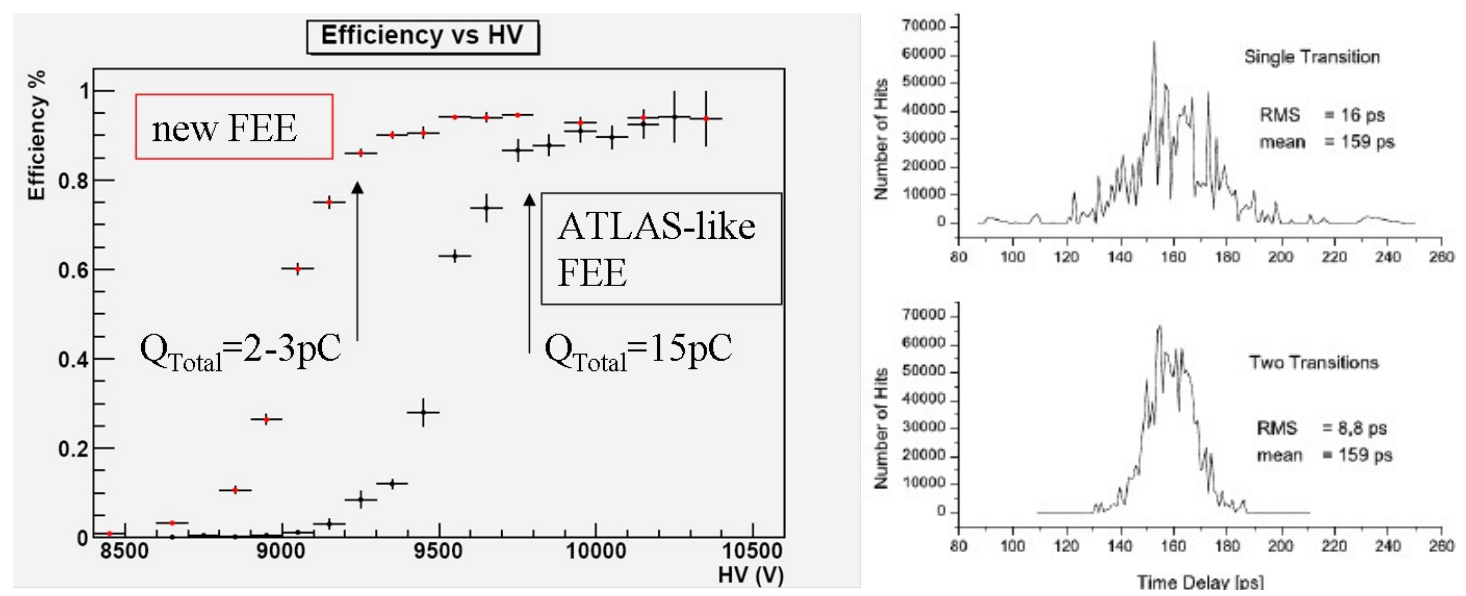

Figure 5: Left: comparison between an ATLAS-like FEE and the newly developed one. The operating charge can be reduced by a factor 5-10. Right: Sub-10ps time resolution obtained with an FPGA-based design, after processing 2 digital signals created by a fast pulse generator [58]. 


\section{Status and future of simulations}

\subsection{The hydrodinamic model}

A simulation mile-stone was achieved in 2004 with the description of the avalanche evolution in Resistive Plate Chambers under its own Space-Charge, largely utilizing first principles [39]. The simulations resorted to the minimal framework for realism, the 1.5D hydrodynamic model $[39,59]$, that allows for a realistic calculation of the electric field during the avalanche evolution, yet within a reasonable computation time. In order to account for the fluctuations in the multiplication process, the equations of evolution were solved in Monte Carlo fashion through a Particle In Cell (PIC) -like procedure. Thanks to improvements in the parameters of the gas, the simulations convincingly explained both the high experimental efficiencies and the charge spectra, that turns out to be completely dominated by the SpaceCharge dynamics. Despite the success of the 1.5D model, a discrepancy up to a factor of 2 was observed in the description of the charge spectra (either prompt or total) while the parameters driving the electronics response function were tuned to describe the observed efficiency and time resolution data, albeit under not unreasonable assumptions. Importantly, much simpler descriptions based on 1D hydro-dynamic models achieve, overall, a higher level of accuracy; they require, however, of one extra parameter to be adjusted, namely, the number of carriers for which space-charge effectively sets in $[60,61]$. On the opposite end, first principle 2D or 3D hydrodynamic MC simulations increase the computation time dramatically and are not widespread (for a comparison between $1.5 \mathrm{D}$ and $2 \mathrm{D}$ see [39]).

Thus, how to achieve a higher descriptive (and predictive) power with potential to boost the parallel-plate technology further?. From the point of view of the avalanche dynamics alone, a natural step is to follow up with the hydro-dynamic approach in the $1.5 \mathrm{D}$ or even $2 \mathrm{D}$ scenario, where the computational overhead might still be acceptable for detector studies. But first, a thorough revision of the parameters of the swarm is pertinent. Early estimates from the Magboltz code (circa 2004), that is generally acknowledged to represent the state of the art for gas detector simulations, differed by as much as $20 \%$ from the recently measured values for the main RPC gas, $\mathrm{C}_{2} \mathrm{H}_{2} \mathrm{~F}_{4}$ [62], most dramatically in the case of the effective Townsend coefficient (see [61] for a comparison). According to [63], latest versions of Magboltz (beyond 8.X.X) have been tuned in order to describe the systematics measured in [62]; unfortunately the agreement does not seem to be satisfactory. It will be very interesting to see how the conclusions of previous simulation works are modified with much more precise parameters of the swarm, at least for a $\mathrm{C}_{2} \mathrm{H}_{2} \mathrm{~F}_{4}$ - only scenario.

Importantly, the ultimate technological limits of the parallel plate technology are connected to the formation of streamers at the highest fields. Although in RPCs they do not manage to trigger breakdown, a deterioration of the time resolution is routinely observed when they are present in a sizable fraction, besides the consequent deterioration in rate capability. Part 
of the observed effect is certainly coming from the presence of precursors. Given the complexity of the streamer phenomena, only 1D hydro-dynamic continuous models have been used so far for describing the behavior of streamers in gaseous detectors [64]. A very qualitative application of this model to RPCs is available, through [65], where indeed the experimental observation of streamer-precursors (for instance, [66]) has been reproduced. Further progress on this crucial aspect is connected both to the improvement in the knowledge of critical parameters of the gas (in particular, those connected to photo-production and photo-ionization) as well as the physical framework.

A major shortcoming when resorting to a hydrodynamic model (either in continuous or Monte-Carlo fashion) is the fact that, due to the macroscopic character of the approach, it is necessary to give up any first-principle description of the avalanche statistics (of paramount importance at threshold level). This information is thus largely unknown at the moment both on the experimental and theoretical side. Fortunately, some theoretical guidance on this aspect exists in the avalanche as well as streamer region ([67], [68]).

\subsection{Microscopic and phenomenological models}

A modern approach to the avalanche problem in gaseous detectors would consist in tracking electrons in 3D in Monte Carlo fashion, considering just fundamental processes. Interactions have to be, therefore, evaluated from the energy gained throughout the drift along the local electric field, together with the interaction cross-sections as a function of the former. Simulations have to be performed in connection with a field solver, provided the field needs to be simultaneously evaluated. ${ }^{5}$ In this approach, avalanche statistics emerge naturally, but the fundamental electron cross-sections are still needed. The much increased computation time will require exploring algorithms for parallel processing or introducing some effective/super-particle technique.

Due to its much higher simplicity, computationally-cheap phenomenological descriptions have to be considered (for examples see [70-72]). They prove very useful for experiment optimization and system design (through the sometimes called 'software digitizer' or 'hit producer'). The technological feedback in this case is, however, little. Either scenario, implemented with the necessary degree of detail, might be already sufficient to describe the, as yet, unexplained response to photons [73], neutrons [74] and heavy ions [75, 76].

A last word on microscopic simulation of streamers in RPCs: accomplishing this endeavor seems to be very far in the horizon, from the theoretical as well as from the experimental side, in view of the large number of unknown parameters needed. Obtaining meaningful answers beyond the simple 1D continuous model will be already very challenging and possibly enough for most present purposes.

\footnotetext{
${ }^{5}$ Here the promising open-source neBEM code, integrated in the Garfield package, has to be mentioned [69], although its usage is far from wide-spread.
} 


\subsection{Field solvers and electromagnetic techniques}

Simulations in [39] (and references therein) achieved a great degree of accuracy in the description of the complicated avalanche dynamics, which was crucial at the time, although all the necessary wrapping-up required for properly interpreting the signals registered at the end of the readout chain was treated in a rather simplified way. Fortunately, it is not difficult to improve the situation, for cases where such an additional sophistication is needed: typical RPC dimensions suggest a conceptual separation between avalanche formation and signal readout, so an 'after-burner' based on an induction+transmission+readout model might suit many practical cases, by treating diverse avalanche models on equal foot [55, 61]. This framework, relying on Multi-Conductor Transmission Line theory (MTL) together with a 2D electrostatic solver, can potentially extend the 1.5D hydrodynamic framework introduced in [39] to a much higher level of realism. This is due to its ability to include practical problems connected to the system response like the characteristic impedance matrix and charge sharing phenomena (required for multi-strip/pad response), transmission losses, connections, amplifier and noise, without making critical approximations. In its most simple version, [61], it merely relies on 2D electrostatic parameters (that are generally much faster to obtain than in the 3D case, especially for multistrip counters). Fig. 6 shows, for illustration, a 2D induction calculation from [2] together with a transmission calculation from [55]. The later aims at illustrating the powerful compensation scheme observed in [77] for the case of external excitation in 2-strip RPCs. Both calculations rely on 2D Finite Element Method (FEM) solvers.
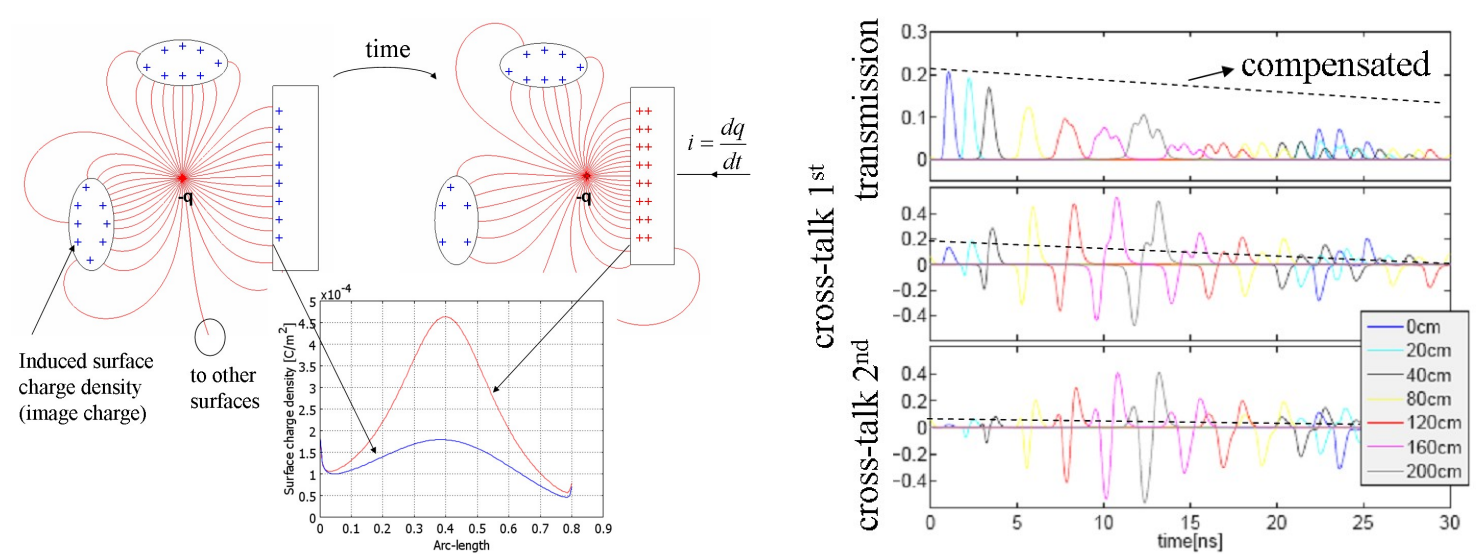

Figure 6: Left: generic induction calculation, showing the field lines and the induced currents. Right: transmission and cross-talk to $1^{\text {st }}$ and $2^{\text {nd }}$ neighbor in a typical $2 \mathrm{~m}$-long RPC for different positions at which the signal has been induced. The dashed lines present the signal levels expected from the compensation technique introduced in [77]. Both simulations rely on 2D FEM solvers.

Finally, when going to a detailed description of the behavior near the counter edges, spacers, but also connections as well as a completely general dynamic response of the RPC itself, it seems unavoidable to use 3D simulations in the long term. An ultimate description of the multi-gap field-equilibration process needs indeed just that, an electro-dynamic (possibly quasi-static) simulation where no assumption is made on the conductivity of the gas, HV- 
coating, resistive plates or the spacers themselves, that would be just included in a generic RPC response function.

\section{Future of classic and multi-gap timing RPCs}

The immediate future of classic RPCs in fundamental research is connected to the sLHC upgrade. The ATLAS upgrade uses a genuine philosophy and one that reflects the unique flexibility of RPCs, thanks in part to the multi-gap approach: a new challenge is addressed with a soft modification of the existing technology, thus largely minimizing possible pitfalls. The bigap extension, together with more sensitive electronics provides just that, an RPC capable of reaching high efficiency at $1 \mathrm{~ns}$ resolution up to $10 \mathrm{kHz} / \mathrm{cm}^{2}$ with a technological design very similar to that of ATLAS. For the CMS upgrade, where requirements are slightly different, there is no decision yet regarding the technological choice.

Among the future experiments relying on classic RPC designs, the Digital Hadron Calorimeter (DHCAL) for the International Linear Collider (ILC) features prominently: its current prototype enjoys almost 500.000 channels. This is allegedly causing a re-birth of digital calorimetry [71, 78]. The DHCAL design has replaced Bakelite by float glass. It will be interesting to see whether a semi-digital (multi-threshold) readout mode can really offer better performances in the challenging saturated-avalanche operation mode, and whether float glass is finally used or Chinese glass is tried instead [79]. Not less impressive than DHCAL is the foreseen 28.800-module magnetized iron neutrino telescope, ICAL, at the Indian-based Neutrino Observatory (INO). Based on classic RPCs also with float glass, it will become the largest RPC-based experiment ever [80]. Year 2012 is very important from the budgetary point of view, so there will be surely important news before celebration of the next workshop.
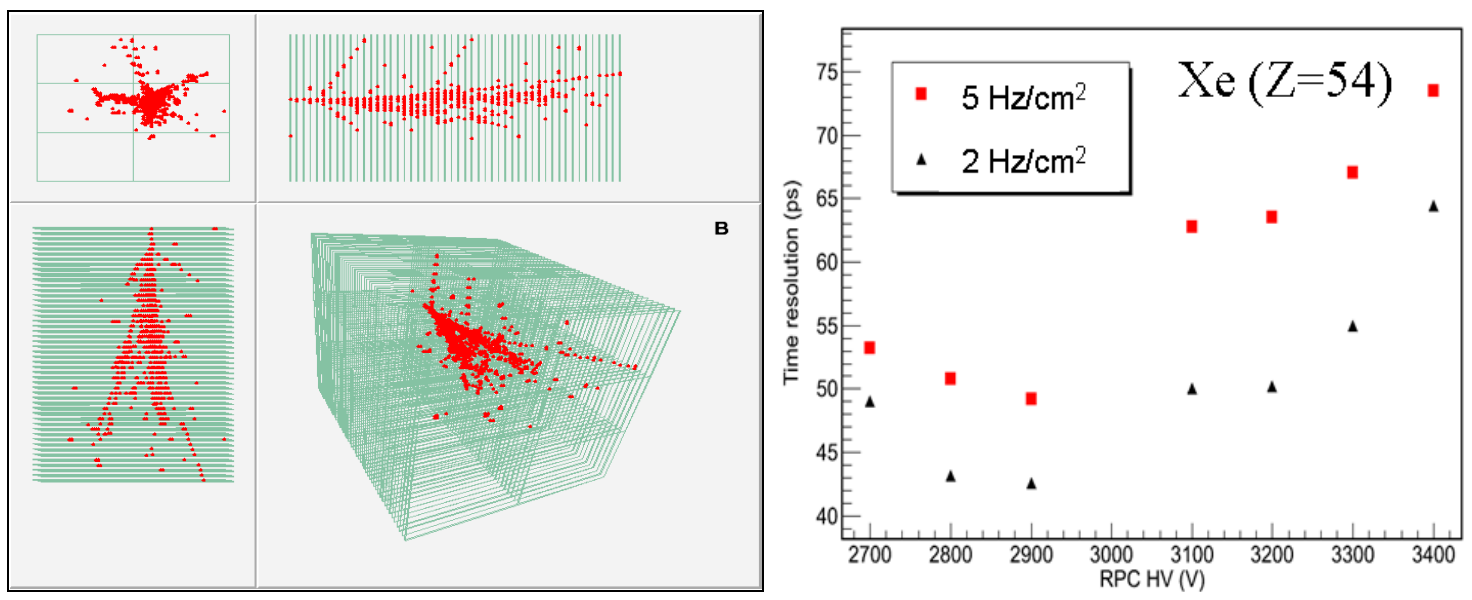

Figure 7: Left: several views of a $16 \mathrm{GeV} / \mathrm{c}$ pion showering on the digital calorimeter developed for the ILC [71]. Right: time response for Xenon ions at different ion flux, as part of the $\mathrm{R}^{3} \mathrm{~B} R \& \mathrm{D}$ (adapted from [76]).

Regarding multi-gap timing RPCs in fundamental physics, further developments are largely circumscribed to the Facility for Antiproton and Ion Research (FAIR), Darmstadt, 
Germany. There, two experiments, the Compressed Baryonic Matter experiment (CBM) as well as $\mathrm{R}^{3} \mathrm{~B}$ (Reactions with Relativistic Radioactive Beams) are planning to make use of MRPC walls. There are many ideas that will be worth following: the Chinese glass -based modules [37, 41], the evolution of the warm glass technique $[81,82]$ and the differential strip readout [83, 84], whose relevance has still to be clarified. It will be also an opportunity to see if it is really possible to obtain good system performances at low levels of inter-strip cross-talk, as some dedicated measurements suggest [77]. This is of paramount importance for the CBM-type heavy ion experiments, where particle multiplicity can be dramatically high. Spurious cross-talk or electromagnetic pick-up can easily jeopardize the challenging 80ps system-resolution that is envisaged [84]. On the other hand, the general-purpose $\mathrm{R}^{3} \mathrm{~B}$ experiment aims at detecting neutrons and heavy ions with two different setups, both with sub-100ps time resolution. Although the first system is almost surely discontinued in virtue of a scintillator calorimeter (see [85], however), it will be very interesting to see the evolution of the latter. Dedicated studies of the detector response under heavy ions are scarce $[75,76,86]$ and they will undoubtedly shed light on the detector behavior and specifically on the Space-Charge phenomena. The time response for the heaviest nuclei studied to date (Xe) is shown in Fig. 7 at different fluxes [76].

Besides FAIR, another facility to look at is Spring, Japan, and in particular the new Laser Electron Photon experiment. Its aim is studying photon-induced hadro-production for which a high-end $50 \mathrm{ps}$ resolution over $5 \mathrm{~m}^{2}$ is required, in order to provide sufficient $\pi / \mathrm{k}$ separation. In view of the low granularity, a strip configuration in two layers seems to comfortably satisfy the requirements, but at the moment all options for the electrode layout seem to be open [87].

\section{New applications}

The field of future applications remains rather constrained to large-area tomography with high position resolution. Applications are largely focused on Positron Emission Tomography (PET), following the pioneering work in [50], and so at present there are several groups involved on different concepts for its optimization $[88,89]$. The RPC-PET makes critical use of the time information in order to improve the position reconstruction along the 2-photon decayline, as well as of the position resolution (in order to reconstruct the position perpendicular to the decay-line) and so it represents an excellent platform for further developing the technology. In this context, understanding the worse resolution obtained for annihilation photons (90-100ps) as compared to minimum ionizing particles will be undoubtedly an important break-through $[73,81]$.

Muon tomography for scanning cargo and general border monitoring of dense materials, on the other hand, is well suited to the RPC technology and can be already accomplished to the accuracy needed with state of the art classic RPCs [90], without resorting to extreme timing capabilities (Fig. 8 right).

A very promising tracking-timing technology was introduced at the workshop [91], by performing timing and tracking with a single detector (better than $100 \mu \mathrm{m} \times 100 \mu \mathrm{m}$ space and 
$100 \mathrm{ps}$ time resolutions were simultaneously achieved). This new idea matches quite naturally the recent proposal for doing 4D-tracking including time information [92]. Although promising, its final relevance for tracking and timing will depend on considerations regarding material budget, system occupancy and specially finding a window of opportunity for the 4D-tracking idea in terms of an adequate physical environment, geometry, and detector performance.

Despite the involvement of several groups in these activities, the impact of RPCs in everyday life is still very limited and new ideas are necessary.

\section{New trends on Resistive Gaseous Detectors}

Resistive Gaseous Detectors quench spark formation down to 'localized discharges' [4], generally believed to be of the streamer-type in most of the so far existing practical applications ([93], for instance). The locality of the process is achieved by limiting the current flow over the electrode surface. There is nothing special on parallel plate geometries that makes them especially suited to this technique except perhaps that they are often forced to work under very higher fields in order to achieve the fastest possible timing response. The growth of solid state alternatives that usually offer a higher reliability as compared to gaseous detectors might explain the changing trend but, either way, some of the high-end micro-patterned gaseous detectors are currently developed following insulating techniques known to the RPC field decades ago. The insulated design offers a much improved detector robustness at the price of a reduced rate capability (due to the charge built-up on the insulator), however a practical compromise can be often achieved.
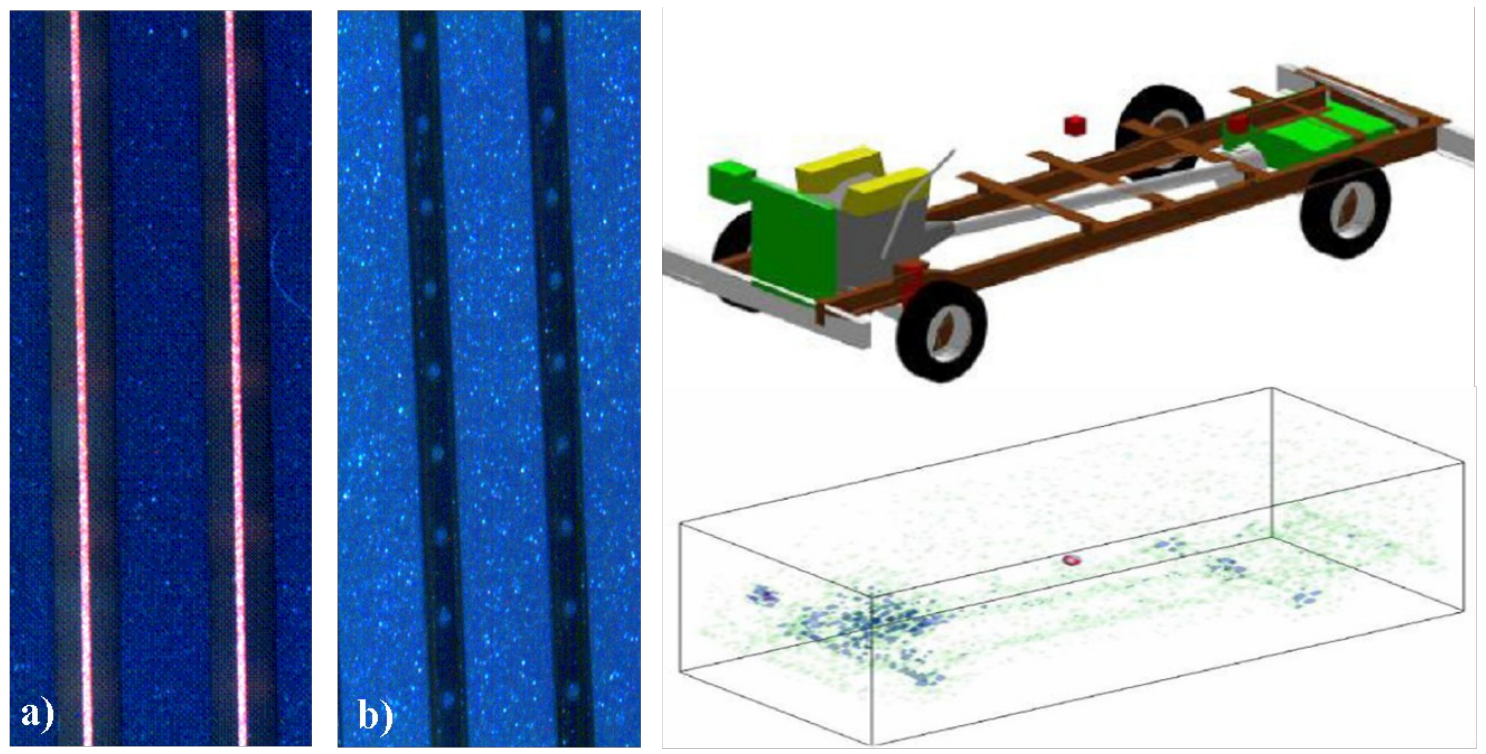

Figure 8: Left: a) A resistive Micro-Strip Gas Detector (anode is conductive) and a fully resistive Micro-Dot Gas Detector (adapted from [94]). Right: simulation results obtained by cosmic muon tomography using RPCs. The $10 \times 10 \times 10 \mathrm{~cm}^{3}$ (red) tungsten block can be found in 1 minute (from [90]). 
Ordinary micro-pattern gaseous detectors (MSGC, Micro-Megas, GEM and others; -see [94] and references therein) are proportional counters offering unprecedented 2D position resolution $(20-40 \mu \mathrm{m})$ and are thus very attractive for many applications: tracking of charged particles, photon-imaging, etc. It has been demonstrated by the RD51 community that the resistive electrode approach can be successfully applied to micro-pattern gaseous detectors and make them robust and spark-protected. Latest designs of resistive micro-pattern detectors have electrodes segmented with resistive strips (Fig.8-left). This approach turns out to be very fruitful and has allowed developing different designs of large-area spark-protected micro-pattern detectors oriented to various applications. Perhaps most remarkably, Micro-Megas with resistive anode strips are envisaged for the $1000 \mathrm{~m}^{2}$ forward wheel upgrade of ATLAS [95]. Since developments aimed at high position resolution are already existing for, so called, microstrip RPCs [96] it seems unavoidable to have in the near future resistive micro-pattern gaseous detectors and genuine RPCs that are functionally equivalent for certain applications. It will be very interesting to see how the technological scenario evolves from that point on.

\section{Conclusions}

We have presented the aims and ambitions of the RPC community towards the next international workshop to be celebrated in Beijing in 2014, and we have done it from the general point of view of resistive gaseous detectors of arbitrary geometry. Many of the goals can be possibly achieved in the short term. Amongst them, the consolidation of already running and future projects, new materials and high-rate techniques together with improved avalanche simulations. Many others, however, like both streamer and generic 3D electro-dynamic simulations, the search for new gases, and the development of general-purpose Front End Electronics, might develop at a slower pace. Above all, the field clearly lives some exciting times at the moment, that we believe will continue materializing in new applications and ideas.

\section{Acknowledgments}

DGD is partially supported by the National Science Foundation of China, under grant 111050110573.

\section{References}

[1] V. Peskov, Challenges for RPCs and resistive micropattern detectors in the next few years, these proceedings.

[2] P. Fonte, Review of RPC simulation and modelling, these proceedings.

[3] Chris Llewellyn Smith, Opinion Piece: The Large Hadron Collider: lessons learned and summary, Phil. Trans. R. Soc. A, 370(2012)995.

[4] The CMS collaboration, Search for the standard model Higgs boson decaying to bottom quarks in pp collisions at $\sqrt{s}_{s}=7 \mathrm{TeV}$, Phys. Lett. B 710(2012)26; The ATLAS collaboration, 
Combined search for the Standard Model Higgs boson using up to $4.9 \mathrm{fb}^{-1}$ of pp collision data at $\sqrt{ }_{S}=7$ TeV with the ATLAS detector at the LHC, Phys. Lett. B 710(2012)49.

[5] V.V. Parkhomchuck, Yu. N. Pestov, N. V. Petrovykh, A spark counter with large area, Nucl. Instr. Meth., 93(1971)269.

[6] R. Santonico, R. Cardarelli, Development of Resistive Plate Counters, Nucl. Instr. Meth. A, $187(1981) 377$.

[7] Archana Sharma, Muon tracking and triggering with gaseous detectors and some applications, Nucl. Instr. Meth. A, 666(2012)98.

[8] E. Cerron Zeballos et al., A new type of resistive plate chamber: The multigap RPC, Nucl. Instr. Meth. A, 374(1996)132.

[9] P. Fonte, A. Smirnitski, M.C.S. Williams, A new high-resolution TOF technology, Nucl. Instr. Meth. A, 443(2000)201.

[10] The CMS collaboration, The CMS experiment at the CERN LHC, JINST, 3(2008)S08004.

[11] The ATLAS collaboration, The ATLAS Experiment at the CERN Large Hadron Collider, JINST, 3(2008)S08003.

[12] A. N. Akindinov et al., Latest results on the performance of the multigap resistive plate chamber used for the ALICE TOF, Nucl. Instr. Meth., A 533(2004)74.

[13] S. An et al., A 20 ps timing device - A Multigap Resistive Plate Chamber with 24 gas gaps, Nucl. Instr. Meth. A, 594(2008)39.

[14] H. R. Band et al., Performance and Ageing Studies of Babar Resistive Plate Chambers, Nucl. Phys. B (Proc. Supp.), 158(2006)139

[15] M. Yamaga et al., RPC systems for BELLE detector at KEKB, Nucl. Instr. Meth. A, $456(2000) 109$.

[16] S. Constantini for CMS, Calibration of the RPC working voltage in the CMS experiment, these proceedings.

[17] G. Chiodini for ATLAS, ATLAS RPC time-of-flight performance, these proceedings.

[18] G. Aielli, at the workshop.

[19] R. Iuppa, Results from the ARGO-YBJ experiment, these proceedings.

[20] A. Paoloni, Performance and ageing of OPERA Bakelite RPCs, these proceedings.

[21] T. Adam et al., Measurement of the neutrino velocity with the OPERA detector in the CNGS beam, arXiv:1109:4897v2[hep-ex].

[22] M. Marchisone, Performance of the ALICE Muon Trigger system in Pb-Pb collisions, these proceedings.

[23] Yi Wang et al., Study on the performance of multi-gap resistive plate chambers, Nucl. Instr. Meth. A, 538(2005)425. 
[24] D. Belver et al., In-beam measurements of the HADES-TOF RPC wall, Nucl. Instr. Meth. A, 602(2009)687.

[25] A. Schuettauf, Timing RPCs in FOPI, Nucl. Instr. Meth. A, 533(2004)65.

[26] V. Ammosov et al., The HARP resistive plate chambers: Characteristics and physics performance, Nucl. Instr. Meth. A, 578(2007)119.

[27] M. Abbrescia, The Extreme Energy Events, these proceedings.

[28] Regulation (EC) No. 842/2006 of the European Parliament and of the Council of 17 May 2006 on certain fluorinated greenhouse gases.

[29] D. Rossi, H. Simon, A closed-circuit gas recycling system for RPC detectors, Nucl. Instr. Meth. 661(2012)145.

[30] R. Cardarelli, V. Makeev, R. Santonico, Avalanche and streamer mode operation of resistive plate chambers, Nucl. Instr. Meth. 382(1996)470.

[31] M. Capeans et al., Optimization of a closed-loop gas system for the operation of Resistive Plate Chambers at the Large Hadron Collider experiments, Nucl. Instr. Meth. A 661(2012)214.

[32] A. Musso, at the workshop.

[33] M. Abbrescia, New gas mixtures for Resistive Plate Chambers operated in avalanche mode, Nucl. Instr. Meth. 661(2012)190.

[34] L. Lopes et al, Systematic study of gas mixtures for timing RPCs, Nucl. Instr. Meth. $661(2012) 194$.

[35] Huangshan Chen et al., R\&D and mass production of LMRPC modules for the STAR-MTD system, these proceedings.

[36] Junjie Zhu, The RPC-based proposal for the ATLAS forward muon trigger upgrade, these proceedings.

[37] J. Wang et al., Development of multi-gap resistive plate chambers with low-resistive silicate glass electrodes for operation at high particle fluxes and large transported charges, Nucl. Instr. Meth. A, 621(2010)151; Y. Wang et al., Aging test of high rate MRPCs, these proceedings.

[38] S. Gramacho, L. Lopes et al., Quantification and inhibition of the gas polymerization process in timing RPCs, Nucl. Instr. Meth. A, 661(2012)194.

[39] C. Lippmann, W. Riegler, Space charge effects in Resistive Plate Chambers, Nucl. Instr. Meth. A, 517(2004)54.

[40] C. Pecharroman, Modeling the RPC behavior from an ion conductivity approach, talk at the X Workshop for Resistive Plate Chambers and related detectors, 2010, Darmstadt, Germany.

[41] J. Wang, Progress on the real-size high-rate MRPC modules \& Conceptual design of the CBM-TOF wall, these proceedings.

[42] A. Laso, Ceramic Resistive Plate Chambers for High Rate Environments, these proceedings. 
[43] M. Abbrescia, The dynamic behaviour of Resistive Plate Chambers, Nucl. Instr. Meth. A, $533(2004) 7$.

[44] D. Gonzalez-Diaz et al., An analytical description of rate effects in timing RPCs, Nucl. Phys. B (Proc. Suppl.), 158(2006)111.

[45] D. Gonzalez-Diaz et al., The role of the Resistive Plate response function in bringing an RPC to an stationary situation, Nucl. Instr. Meth. A, 602(2009)713.

[46] C. Lippmann et al., Rate effects in resistive plate chambers, Nucl. Phys. B (Proc. Suppl.), 158(2006)128.

[47] B. Bilki et al., Measurement of the rate capability of Resistive Plate Chambers, JINST(2009) 4, P06003.

[48] M. Morales, Aging and conductivity of electrodes for high rate tRPCs from an ion conductivity approach, these proceedings.

[49] P. Fonte et al., A spark-protected high-rate detector, Nucl. Instr. Meth., 431(1999)154.

[50] A. Blanco et al., RPC-PET: A New Very High Resolution PET Technology, IEEE Trans. Nucl. Sci. 53, 5(2006)2489.

[51] F. Anghinolfi et al., NINO: an ultra-fast and low-power front-end amplifier/discriminator ASIC designed for the multigap resistive plate chamber, Nucl. Instr. Meth. 533(2004)183.

[52] M. Ciobanu et al., PADI: a fast Preamplifier-Discriminator for Time-of-Flight Measurements. IEEE (NSSC conference), N30-18(2008).

[53] X. Zhou, CAD: A Current-Mode Amplifier and Discriminator ASIC for MRPC-TOF Detectors, these proceedings.

[54] R. Santonico, at the workshop.

[55] D. Gonzalez-Diaz, RPC simulations from a current stand-point, these proceedings.

[56] L. Paolozzi, Test for upgrading the RPCs at very high counting rate, these proceedings.

[57] http://tdc.web.cern.ch/TDC/hptdc/hptdc.htm, accessed 26-March-2012.

[58] G. Korcyl, TRB3- multichannel TDC and DAQ platform, these proceedings.

[59] K. Doroud et al., Recombination: An important effect in multigap resistive plate chambers, Nucl. Instr. Meth. A, 610(2009)649.

[60] M. Abbrescia et al., Resistive Plate Chambers in avalanche mode: a comparison between model predictions and experimental results, Nucl. Instr. Meth. A, 409(1998)1.

[61] D. Gonzalez-Diaz, Simulation of resistive plate chambers with multi-strip readout, Nucl. Instr. Meth. A, 661(2012)172.

[62] J. de Urquijo et al., Electron swarm coefficients in 1,1,1,2 tetrafluoroethane (R134a) and its mixtures with Ar, Eur. Phys. J. D 51(2009)241.

[63] S. Biaggi, private communication. 
[64] P. Fonte, A model of breakdown in parallel-plate detectors, IEEE Trans. Nucl. Sci. 43(1996)2135.

[65] A. Moshaii et al, RPC simulation in avalanche and streamer modes using transport equations for electrons and ions, Nucl. Instr. Meth. 661(2011)470.

[66] P. Fonte, V. Peskov, F. Sauli, Feedback and breakdown in parallel plate chambers, Nucl. Instr. Meth. 305(1991)91.

[67] W. Legler, Die Statistik der Elektronenlawinen in elektronegativen Gasen, bei hohen Feldstarken und bei grosser Gasverstarkung, Z. Naturforschung 16a(1961)253.

[68] A. Mangiarotti et al., On the deterministic and stochastic solutions of Space Charge models and their impact on high resolution timing, Nucl. Phys. B (Proc. Suppl.), 158(2006)118.

[69] N. Majumdar et al., A Computation of $3 D$ electrostatic weighting field in Resistive Plate Chambers, Nucl. Instr. Meth. A, 595(2008)346.

[70] M. Roeder, A $2 m \times 0.5 m$ prototype of MRPC-based neutron detector with steel converter plates, at the workshop.

[71] J. Repond, Analysis of DHCAL events, these proceedings.

[72] D. Gonzalez-Diaz, Research and developments on timing RPC's. Application to the ESTRELA detector of the HADES experiment at GSI, JINST TH003.

[73] C. Lippmann et al., Simulation of RPC performance for $511 \mathrm{keV}$ photon detection, Nucl. Instr. Meth. A, 602(2009)735

[74] C. Caesar et al., NeuLAND MRPC-based detector prototypes tested with fast neutrons, Nucl. Instr. Meth. A, 661(2012)145.

[75] P. Cabanelas et al., Performances of 4-gap timing RPCs for relativistic ions in the range $Z=1-6, \operatorname{JINST}(2009)$ 4, P11007.

[76] C. Paradela, Performance of timing-RPC prototypes with relativistic heavy ions, these proceedings.

[77] D.Gonzalez-Diaz et al., Signal coupling and signal integrity in multi-strip resistive plate chambers used for timing applications, Nucl. Instr. Meth. A, 648(2011)52.

[78] L. Xia., Construction of a Digital Hadron Calorimeter with Resistive Plate Chambers, these proceedings.

[79] I. Laktineh, Development of large GRPC for a Semi-Digital Hadronic Calorimeter for $I L C$, these proceedings.

[80] S. Dasgupta, Proposed trigger scheme for the ICAL detector of the India based Neutrino Observatory, these proceedings.

[81] D. Gonzalez-Diaz et al., The effect of temperature on the rate capability of timing RPCs, Nucl. Instr. Meth. A, 555(2005)72. 
[82] I. Deppner, A multi-strip MRPC prototype for the CBM time-of-flight wall, these proceedings.

[83] M. Petrovici, High counting rate, differential. strip read-out, multi-gap, timing RPC, these proceedings.

[84] I. Deppner et al., The CBM time-of-flight wall, Nucl. Instr. Meth. A, 661(2012)121.

[85] J. Machado, Simulations of an innovative Time-of-Flight Detector for high energy neutrons based on iron-less RPCs, these proceedings.

[86] E. Casarejos et al., Detection efficiency of relativistic heavy-ions with resistive-plate chambers, Nucl. Instr. Meth. A, 674(2012)39.

[87] N. Tomida, Design and performance of large area, high resolution RPCs for LEPS2 at SPring-8, at the workshop.

[88] K. Doroud et al., MRPC-PET: A new technique for high precision time and position measurements, Nucl. Instr. Meth. A, 660(2011)73.

[89] B. Pavlov, A multi-gap RPC based detector for gamma rays, these proceedings.

[90] P. Baesso, Muon scattering tomography with resistive plate chambers, these proceedings.

[91] P. Fonte, TOFtracker: combination of time-of-flight and high-accuracy bi-dimensional tracking in a single gaseous detector, these proceedings.

[92] J. A. Garzon et al., TimTrack: A new concept for the tracking of charged particles with timing detectors, Nucl. Instr. Meth. A, 629(2011)311.

[93] U. Denni et al., Streamer studies in Resistive Plate Chambers, Nucl. Instr. Meth. A, 640(2011)76.

[94] Vladimir Peskov, Resistive microstrip and microdot detectors: a novel approach in developing spark protected micropattern detectors, these proceedings.

[95] M. Byszewski, Development of spark-resistant Micro-Megas with resistive anode strips, these proceedings.

[96] T. Francke et al., Potential of RPCs for tracking, Nucl. Instr. Meth. A, 508(2003)83. 